\title{
Teleodontologia em disfunção temporomandibular e dor orofacial durante a pandemia de COVID-19: relato de caso
}

Using teledentistry in temporomandibular dysfunction and orofacial pain during COVID-19 pandemic: case report

Teleodontología en disfunción temporomandibular y dolor orofacial durante la pandemia COVID-19: reporte de caso

Thiago Medina BRAZOLOTO ${ }^{1}$

Fabio José Condino FUJARRA ${ }^{2}$

Ana Paula de LIMA ${ }^{2}$

Angélica Duarte Coelho CAMARGO ${ }^{2}$

Luciana Lima da Silva FREITAS ${ }^{2}$

Flávia Cristina Cremonesi CARDOSO²

${ }^{I}$ Oficial Dentista da Polícia Militar do Estado de São Paulo, Professor da Faculdade de Medicina da Universidade Cidade de São Paulo (UNICID), 01106-000, São Paulo - SP, Brasil

${ }^{2}$ Oficial Dentista da Polícia Militar do Estado de São Paulo

\section{Resumo}

A pandemia do novo coronavírus provocou a suspensão temporária de atendimento odontológico eletivo a fim de diminuir a circulação de pessoas e de procedimentos odontológicos que possam favorecer disseminação do vírus. Pacientes que apresentam sinais e sintomas dolorosos ou incapacitantes, tais como dor orofacial ou disfunção temporomandibular, devem ter seus atendimentos de urgência e emergência garantidos. Este cenário fez da teleodontologia uma necessidade para ampliar o acesso ao doente com maior segurança, além de uma oportunidade para planejar estratégias de atendimento presencial. Especialmente para a assistência à saúde no âmbito militar, a teleodontologia pode ser uma ferramenta muito útil para oferecer apoio e mecanismos de assistência à saúde, ao mesmo tempo em que mantém o profissional da área de segurança pública trabalhando, além de outras vantagens. O objetivo deste trabalho é descrever dois atendimentos em teleodontologia em dor orofacial no âmbito militar e discutir a atuação profissional à distância e a experiência de um serviço especializado durante a pandemia do novo coronavírus.

Descritores: Teleodontologia; Infecções por Coronavirus; Neuralgia do trigêmeo; Dor Facial; Síndrome da Disfunção da Articulação Temporomandibular; Odontologia Militar.

\section{Abstract}

The pandemic of the new coronavirus caused the temporary suspension of elective dental care in order to decrease the circulation of people and dental procedures that may favor the spread of the virus. Patients, who present painful or disabling signs and symptoms, as in cases of orofacial pain or temporomandibular disorder, must have their urgency and emergency care guaranteed. For these reasons teledentistry became necessary to increase access to the patient with security, in addition to an opportunity to plan strategies for face-to-face appointment. Especially for health care in the military field, teledentistry can be a very useful tool to offer support and health care mechanisms, while keeping the public safety employees working, in addition to other advantages. The aim of this paper is to describe two case reports in teledentistry in orofacial pain in the military sphere and to discuss the remote dental care and the experience of an expert service during the pandemic of the new coronavirus.

Descriptors: Teledentistry; Coronavirus Infections; Trigeminal Neuralgia; Facial Pain; Temporomandibular Joint Dysfunction Syndrome; Military Dentistry.

\section{Resumen}

La pandemia del nuevo coronavirus provocó la suspensión temporal de la atención odontológica electiva con el fin de reducir la circulación de personas y los procedimientos dentales que pueden favorecer la propagación del virus. Los pacientes que presenten signos y síntomas dolorosos o incapacitantes, como en los casos de dolor orofacial o trastornos temporomandibulares, deben tener garantizada su atención de urgencia y emergencia. Este escenario hizo de la teleodontología una necesidad para incrementar el acceso al paciente con mayor seguridad, además de una oportunidad para planificar estrategias de atención presencial. Especialmente para la asistencia sanitaria en el ámbito militar, la teleodontología puede ser una herramienta muy útil para ofrecer mecanismos de apoyo y asistencia sanitaria, manteniendo en funcionamiento al profesional de la seguridad pública, además de otras ventajas. El objetivo de este trabajo es describir dos reportes de casos en teleodontología en dolor orofacial en el ámbito militar y discutir el desempeño profesional a distancia y la experiencia de un servicio especializado durante la pandemia del nuevo coronavirus.

Descriptores: Teleodontología; Infecciones por Coronavirus; Neuralgia del Trigémino; Dolor Facial; Síndrome de la Disfunción de Articulación Temporomandibular; Odontología Militar.

\section{INTRODUÇÃO}

O surgimento do novo coronavírus (SARSCoV-2) e a doença por ele provocada (COVID-19), com os primeiros registros em dezembro de 2019, na cidade de Wuhan, na China, tem trazido grande impacto socioeconômico à população mundial. Especialmente na assistência à saúde, muitos serviços eletivos foram interrompidos ${ }^{1}$, a fim de minimizar a circulação de pessoas e a transmissão do vírus e de priorizar mais leitos hospitalares e profissionais da saúde aos doentes da COVID-192, o que traz uma preocupação acerca do aumento do risco para outras doenças bucais e sistêmicas ${ }^{3}$, especialmente em idosos ${ }^{4}$. Em Odontologia, a rota de transmissão do novo coronavírus tem importante envolvimento na prática clínica, pois muitos procedimentos odontológicos produzem aerossol que pode estar contaminado dessas partículas virais, facilitando seu espalhamento, motivo pelo qual diversas medidas de biossegurança devem ser, rigorosamente, adotadas ${ }^{2}$. Desta forma, o adiamento dos tratamentos odontológicos eletivos faz com que a teleodontologia e as ferramentas de telecomunicação sejam promissoras para manter o contato com o paciente sem colocá-lo em alto risco de infecção ${ }^{5}$.

$\mathrm{O}$ uso de tecnologias da informação e da comunicação aplicadas à saúde, seja em educação a distância, em pesquisa ou em assistência em saúde está relacionado ao aumento da demanda por telessaúde, incluindo telemedicina e teleodontologia, o que traz grande impacto positivo na resolutividade na assistência à saúde, no baixo custo e na educação continuada de profissionais da saúde ${ }^{6}$. Nesta situação 
de grandes limitações, o acompanhamento profissional a distância em teleconsultas pode ajudar no tratamento e a estabelecer um relacionamento profissional-paciente mais forte ${ }^{7}$. Neste campo, a telessaúde é uma estratégia de saúde digital para o Brasil que, conforme decreto federal $\mathrm{n}^{\circ} 9.795$, de 17 de maio de 2019, deve trazer benefícios à população como a expansão e melhoria da rede de serviços de saúde ${ }^{8}$.

Especula-se que o isolamento social durante a pandemia de COVID-19 possa causar impacto psicológico negativo, com possível aumenta do risco de desenvolvimento, piora ou perpetuação de bruxismo e disfunção temporomandibular, motivo pelo qual os profissionais devem estar atentos aos sinais e sintomas para o manejo adequado, bem como ao uso da teleodontologia .

O objetivo deste trabalho foi descrever dois atendimentos em teleodontologia em dor orofacial no âmbito militar e discutir a atuação profissional a distância e a experiência de um serviço especializado durante a pandemia do novo coronavírus.

\section{CASO CLÍNICO}

\section{- Caso Clínico 1}

Paciente J.R.I.P., gênero masculino, 47 anos, pardo, policial militar, compareceu ao cirurgiãodentista (CD) clínico-geral da Unidade Integrada de Saúde da Polícia Militar, localizada em Taubaté/SP, após encaminhamento do médico otorrinolaringologista, com queixa de paralisia facial à esquerda e dor na face do lado direito que limitava a abertura bucal, além de dificuldade de selamento palpebral. Foi encaminhado com urgência ao Centro Odontológico da Polícia Militar do Estado de São Paulo (CODONT), na cidade de São Paulo. Relatou que uma semana antes teve sensação de "ouvido tapado" (plenitude auricular) e de pressão na orelha esquerda; ao $3^{\circ}$ dia sentiu dormência e paralisia na hemiface esquerda. Não havia histórico recente de infecções virais, traumatismos locais, contato com animais, exceto cão, que não possuía carrapatos. Fez uso de betametasona, com melhora da plenitude auricular. Informou ter hipertensão arterial sistêmica e diabetes mellitus tipo II. Em uso de losartana (100 $\mathrm{mg} / \mathrm{dia})$ e metformina (1000 $\mathrm{mg} / \mathrm{dia})$, rosuvastatina $(20 \mathrm{mg} / \mathrm{dia})$. Negou alergias. Ao exame físico: hipomotricidade facial à esquerda, teste de sensibilidade tátil realizado com filamentos de von Frey evidenciou hipoestesia tátil fina em dermátomos trigeminais oftálmico e mandibular esquerdos; sem lesões de cárie dentária; doença periodontal crônica generalizada controlada, língua edentada, sem sinais de atrição dentária; estalido em ATM esquerda (início de abertura e final de fechamento bucais), em ATM direita (final da abertura bucal) e subluxação de ATM direita; à palpação muscular, dor intensa em mm. masséteres $(\mathrm{D}+/ \mathrm{E}+++), \mathrm{mm}$. temporais (terços anteriores) $(\mathrm{D}+/ \mathrm{E}+++)$, ângulo mandibular esquerdo $(++)$, assoalho bucal (área submandibular) $(\mathrm{E}++)$, $\mathrm{mm}$. cervicais $(\mathrm{E}+)$; saliva com aspecto normal durante ordenha da glândula parótida esquerda. Exame da radiografia panorâmica: ATM normais, alongamento processos estiloides, perda óssea horizontal alveolar. Hipóteses Diagnósticas: Paralisia Facial de Bell. Periodontite. Bruxismo do sono. Dor miofascial. Conduta: Dexametasona $4 \mathrm{mg}$ ( $1^{\circ}$ dia $)$, $2 \mathrm{mg}\left(2^{\circ} \mathrm{dia}\right), 1 \mathrm{mg}\left(3^{\circ}\right.$ e $4^{\circ}$ dias), citoneurin 5000 (drágeas) a cada 8 horas por 7 dias, cloridrato de ciclobenzaprina $5 \mathrm{mg}$ por 7 noites; medidas de terapia física (calor) em face à esquerda e exercícios de motricidade facial e mandibular. Em face da pandemia do novo coronavírus e do estado de quarentena, o paciente compareceu à $\mathrm{CD}$ clínicageral e optamos pelo atendimento à distância (teleodontologia) (Figura 1). A experiência da odontologia militar e do cenário de pandemia ampliou os aspectos a serem analisados para elegibilidade da teleodontologia em nosso serviço especializado (Figura 2).

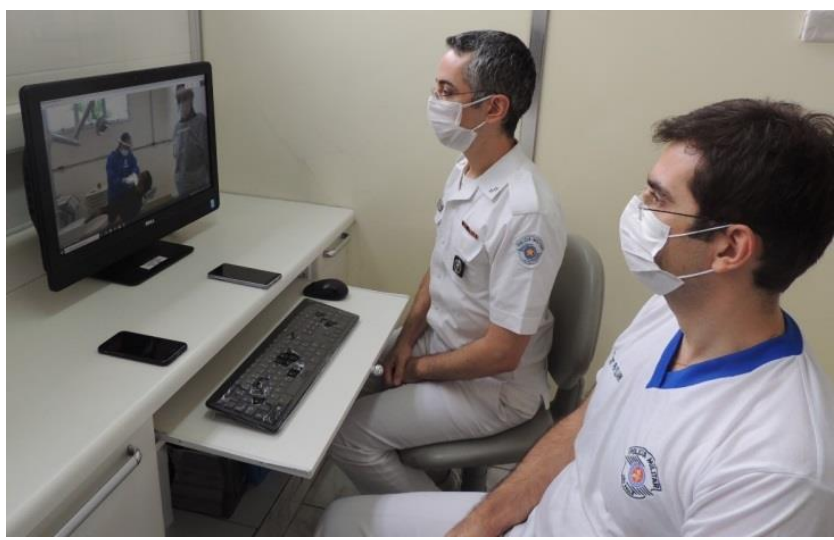

Figura 1: Atendimento clínico com supervisão em videoconferência (teleodontologia).

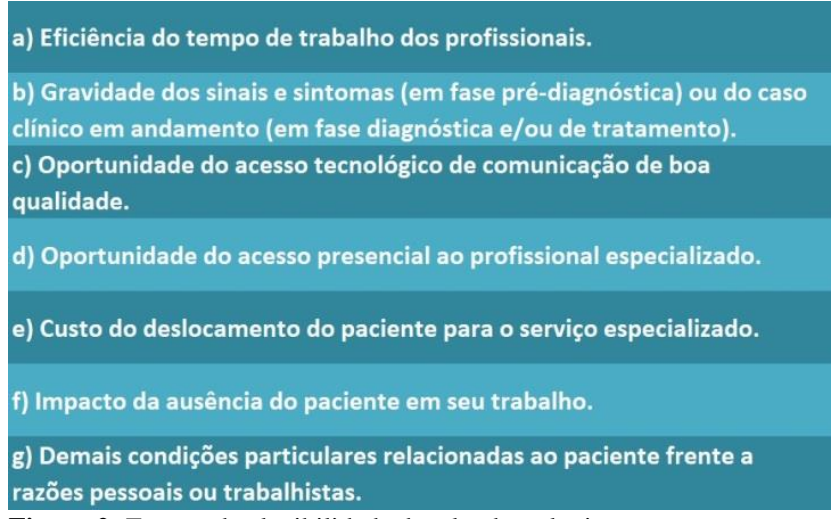

Figura 2: Fatores de elegibilidade da teleodontologia

- Primeira Videoconferência: após 1 semana, compareceu ao $\mathrm{CD}$ clínico-geral que realizou a primeira consulta por meio de videoconferência com o CD especialista. Houve melhora na dor, mas havia queixa de limitação na abertura bucal e selamento palpebral e ainda apresentava dificuldade motora em hemiface esquerda. Queixa de dor ao engolir e ardência no palato. Ao exame físico, o clínico-geral 
observou eritema em palato, sem placa branca. Hipótese diagnóstica: candidose eritematosa atrófica. Conduta: nistatina 100.000 UI, solução oral, bochechos de $5 \mathrm{ml}$ por 3 a 5 min, 5 vezes ao dia por 7 dias; mantidos citoneurin 5000 a cada 8 horas e cloridrato de ciclobenzaprina $5 \mathrm{mg} /$ noite por 7 dias, bem como exercícios mandibulares e medidas físicas em face.

- Segunda Videoconferência: após quinze dias do início do tratamento, paciente relatou melhora significativa dos sintomas, ausência de dor, discreta assimetria facial e fechamento palpebral com leve dificuldade, além de melhora na motricidade facial e ausência de lesões orais. Conduta: mantidos exercícios faciais e mandibulares.

- Terceira Videoconferência: após vinte e dois dias do início do tratamento, todas as queixas do paciente foram solucionadas. Conduta: solicitada tomografia computadorizada com contraste do crânio, a fim de investigar lesões no Sistema Nervoso Central (SNC). Após cerca de um mês, retornou ao CD clínico-geral com a tomografia com características dentro da normalidade, bem como bom estado geral diante de avaliação médica.

\section{- Caso Clínico 2}

Paciente R.A.M., gênero masculino, 44 anos, pardo, policial militar, músico, paciente regular do ambulatório de Dor Orofacial do CODONT, com diagnóstico de neuralgia trigeminal e em investigação diagnóstica para descartar afecções no sistema nervoso central, seguia realizando exames complementares e em uso de carbamazepina (400mg/dia) quando adveio a quarentena imposta pela pandemia de COVID-19. Até então, o caso não apresentava andamento satisfatório em razão do atraso do paciente em providenciar os exames solicitados e da distância física entre a residência do doente e o ambulatório especializado, que o levava a postergar as consultas de acompanhamento. A partir da pandemia, uma única consulta de urgência fora realizada presencialmente no CODONT, em virtude de uma crise de dor latejante, contínua, de intensidade moderada na região da hemiface direita e dentes ipsilaterais, que se sobrepunha aos paroxismos desta região e que, aparentemente, os agravava. Naquela oportunidade, o exame intra-oral revelou periodontite na raiz mesial do dente 48. Conduta: tratamento periodontal, carbamazepina $(600 \mathrm{mg} / \mathrm{dia})$. As demais a ações clínicas para diagnóstico e tratamento passaram a ser realizadas por teleodontologia, reunindo paciente e CD clínico-geral radicados na Unidade Integrada de Saúde da Polícia Militar, localizada em São José dos Campos/SP, e CD especialista na capital paulista.

- Primeira Videoconferência: relatou 60\% de melhora do quadro global e $30 \%$ em relação à última consulta. Ao exame: sem dor à palpação de músculos mastigatórios, sem alodínia em face ou dor à deglutição; zona gatilho intra-oral em mucosa vestibular entre os dentes 48 e 45, inativada pela infiltração anestésica local. Conduta: carbamazepina $800 \mathrm{mg} / \mathrm{dia}$, bochechos com solução de cloridrato de benzidamina $1,5 \mathrm{mg}$ antes das refeições; novos exames laboratoriais foram solicitados para avaliação da função hepática.

- Segunda Videoeconferência: após trinta dias, o paciente evoluiu com piora do quadro clínico, tanto na intensidade quanto na frequência de dores paroxísticas com impacto negativo sobre sua qualidade de vida e sua alimentação; gamaglutamiltransferase aumentou para 136U/L. Conduta: retirada gradual da carbamazepina e sua substituição por oxicarbazepina $(600 \mathrm{mg} / \mathrm{dia})$; encaminhamento ao neurologista. Após sete dias, em consulta com CD clínico-geral, apresentou melhora expressiva da dor e de sua qualidade de vida.

DISCUSSÃO

Em 16 de março 2020, o Conselho Federal de Odontologia (CFO) junto ao Ministério da Saúde recomendou a suspensão de atividades odontológicas eletivas no sistema público de saúde, a fím de contribuir para a contenção do avanço do novo coronavírus, por evitar a contaminação cruzada ${ }^{10}$. Na área médica, o Conselho Federal de Medicina (CFM) reconheceu junto ao Ministério da Saúde em 19 de março 2020 a possibilidade e a eticidade da utilização da telemedicina durante a pandemia, para aplicação na modalidade de: a) teleorientação: para que profissionais da medicina realizem à distância a orientação e o encaminhamento de pacientes em isolamento; b) telemonitoramento: ato realizado sob orientação e supervisão médica para monitoramento à distância de parâmetros de saúde e/ou doença; c) teleinterconsulta: exclusivamente para troca de informações e opiniões entre médicos, para auxílio diagnóstico ou terapêutico ${ }^{11}$. Em 04 de junho de 2020, o CFO publicou a resolução CFO-226 ${ }^{12}$, que disciplinou o exercício da Odontologia a distância em razão da pandemia de SARS-CoV-2, permitindo que o CD assistente troque informações e opiniões com outro CD, com o objetivo de prestar uma melhor assistência ao paciente ${ }^{12}$. Ainda, permite a teleodontologia em duas modalidades: 1) telemonitoramento, em que o $\mathrm{CD}$ pode realizar o acompanhamento a distância dos pacientes que estejam em tratamento, no intervalo ou período entre uma consulta e outra; 2) teleorientação em que o CD pode apenas identificar, através da realização de questionário pré-clínico, o melhor momento para a realização do atendimento presencial. Por outro lado, antes da referida resolução CFO-226, o código de ética odontológica já previa a relação de discussão ou análise de casos clínicos entre profissionais, conforme artigo 25: "Para fins de diagnóstico e tratamento o especialista poderá conferenciar com 
outros profissionais" $"$. Nos casos aqui apresentados, parece-nos adequado denominar os atendimentos a distância como teleconsulta supervisionada, o que se aproxima das modalidades teleinterconsulta, na medicina, e telemonitoramento, na odontologia, embora tenha sido realizada, de fato, uma consulta odontológica na presença do clínico-geral e supervisão em tempo real do especialista a distância. Todavia, trata-se de uma consulta com o especialista, mediada pelo clínico-geral que colabora para anamnese e fornece subsídios de exame físico, orientado pelo especialista para que este possa formar as hipóteses diagnósticas e indicar as condutas, após a discussão e concordância entre os profissionais. Claramente, a resolução CFO-226 trouxe um detalhamento maior sobre a atuação odontológica à distância, a fim de ampliar o alcance da população a informações mais seguras sobre $\mathrm{o}$ atendimento odontológico, bem como a melhor qualidade da informação quando da assistência profissional local, além de coibir condutas antiéticas e prejuízos à saúde pública. Por outro lado, as características do atendimento aqui relatadas parecem ampliar esta atuação de forma ética.

Há uma falta de conhecimento entre os cirurgiões-dentistas sobre o que é e de que forma pode ser exercida a teleodontologia, apesar de entenderem sua importância e terem uma postura positiva em relação ao tema. Isso evidencia a necessidade programas que favoreçam esta prática, como o reconhecimento de avanços tecnológicos, a inclusão da teleodontologia na prática diária nas diversas especialidades e o apoio da comunidade profissional ${ }^{14}$. Por isso, há grande necessidade de melhorar o conhecimento e promover a teleodontologia junto a toda comunidade profissional. Isso pode ser alcançado por meio de programas de educação continuada e campanhas de conscientização para os profissionais, a fim de levar a uma melhoria na oferta de cuidados à saúde oral a populações remotas ${ }^{15}$

Em pesquisa que avaliou a aplicabilidade da teleodontologia em tempo real, por videoconferência em âmbito militar, para fins de inspeção da cavidade oral por meio de câmera intraoral, concluiu que o método se mostrou efetivo, seguro e viável, com vantagem econômica, menor risco inerente ao deslocamento do paciente e menor tempo gasto com deslocamentos, disponibilizando o policial militar para sua atuação em segurança pública ${ }^{16}$.

$\mathrm{O}$ uso de tecnologias para soluções em telemonitoramento pode ampliar a assistência à saúde, especialmente na redução de doenças crônicas e na construção de uma rede integral de atenção básica, trazendo inovações à indústria, redução de custos ao sistema de saúde. Ainda, há uma carência na produção científica no campo específico do telemonitoramento ${ }^{17}$.
A teleodontologia utiliza registros eletrônicos de saúde, tecnologia de telecomunicações, imagem digital e Internet para fornecer educação e consultoria com especialistas em áreas remotas. A teleconsulta pode ocorrer em tempo real, com comunicação direta por videoconferência entre um profissional $(\mathrm{CD}$ ou técnico de saúde bucal) ou paciente em uma comunidade remota e um CD ou especialista em uma comunidade maior que fornece suporte ou supervisão. Também pode ser realizada por registros eletrônicos de saúde e vídeos armazenados e enviados para que especialista emita uma opinião ${ }^{18}$. Porém, critérios como dificuldade de acesso ou o viver em áreas remotas não parecem esgotar as indicações para teleodontologia que se observam atualmente.

A Neuralgia Trigeminal é uma doença que exige investigação diagnóstica com brevidade, já que pode haver lesões tumorais associadas ${ }^{19}$. A distância entre a residência do paciente e o serviço de saúde dificultou e adiou o comparecimento à consulta $\mathrm{e}$ acompanhamento antes da pandemia pelo paciente do caso 2, o qual, por conta própria, interrompeu o uso da medicação prescrita e demorou a realizar o exame subsidiário solicitado. Assim como em outros países, os atendimentos presenciais de urgência $\mathrm{e}$ emergência não foram interrompidos e sofreram adaptações necessárias $^{20}$, o que ocorreu em nosso serviço a fim de prestar o atendimento exequível, frente às limitações da pandemia, o mais breve possível.

Um estudo avaliou que dentre as principais doenças que se beneficiam com o uso das tecnologias de comunicação na teleodontologia, as disfunções temporomandibulares e as dores orofaciais representaram cerca de $45 \%$ das $\operatorname{consultas}^{18}$. A experiência aqui relatada revela que a teleodontologia pode levar o especialista em disfunção temporomandibular e dor orofacial ao clínico-geral por meio de teleconsultas a distância. Estes doentes requerem atendimentos frequentes e podem representar um custo elevado ao sistema de saúde.

\section{CONSIDERAÇÕES FINAIS}

A assistência odontológica na especialidade de Disfunção Temporomandibular e Dor Orofacial deve ser reconhecida e mantida para os casos de urgência durante a pandemia, além do monitoramento dos casos que estavam na vigência de tratamento antes da pandemia. A possibilidade de aumento de casos nesta especialidade, decorrentes da interrupção dos atendimentos eletivos e, portanto, da criação de uma demanda reprimida, sugere a realização de triagem nos serviços de saúde a fim de reconhecer os indicadores epidemiológicos após a pandemia. A teleodontologia se mostra um caminho oportuno, frente ao cenário de pandemia, e necessário para os pacientes que apresentem condições urgentes de 
atendimento presencial, permitindo as devidas orientações de encaminhamento. A teleconsulta supervisionada trouxe economia de tempo e de recursos materiais, além de contribuir para a experiência do clínico-geral sobre aspectos clínicos especializados. Critérios para elegibilidade do atendimento em teleodontologia merecem ser melhor estudados, a fim de otimizar e ampliar as práticas à distância com resultados positivos e promissores para a odontologia, especialmente no âmbito militar, observado o impacto social pela manutenção dos trabalhadores da segurança pública.

\section{REFERÊNCIAS}

1. Naqvi K, Mubeen SM, Ali Shah SM. Challenges in providing oral and dental health services in COVID19 pandemic. J Pak Med Assoc. 2020;70(Suppl 3)(5):S113-17.

2. Meng L, Hua F, Bian Z. Coronavirus Disease 2019 (COVID-19): Emerging and Future Challenges for Dental and Oral Medicine. J Dent Res. 2020;99(5):481-87.

3. Dave M, Seoudi N, Coulthard P. Urgent dental care for patients during the COVID-19 pandemic. Lancet. 2020;395(10232):1257.

4. León S, Giacaman RA. COVID-19 and Inequities in Oral Health Care for Older People: An Opportunity for Emerging Paradigms. JDR Clin Trans Res. 2020;5(4):290-92.

5. Pereira LJ, Pereira CV, Murata RM, Pardi V, Pereira-Dourado SM. Biological and social aspects of Coronavirus Disease 2019 (COVID-19) related to oral health. Braz Oral Res. 2020;34:e041.

6. Haddad AE, Bönecker M, Skelton-Macedo MC. Research in the field of health, dentistry, telehealth and teledentistry. Braz Oral Res. 2014;28:S180683242014000100003

7. Giudice A, Barone S, Muraca D, Averta F, Diodati F, Antonelli A et al. Can Teledentistry Improve the Monitoring of Patients during the Covid-19 Dissemination? A Descriptive Pilot Study. Int J Environ Res Public Health. 2020;17(10):3399.

8. Brasil. DECRETO No 9.795 , DE 17 DE MAIO DE $2019 . \quad$ Disponível em: http://www.planalto.gov.br/ccivil_03/_ato20192022/2019/decreto/D9795.htm. Acessado em: $15 / 06 / 2020$.

9. Oliveira SSI, Gonçalves SLM, Weig KM, Magalhães Filho TR, Martinez OER, Kalil MTAC et al. Temporomandibular disorders: Guidelines and Self-Care for Patients During COVID-19 Pandemic. Braz Dent Sci. 2020 Apr/Jun;23(2, supl):1-8

10. Conselho Federal de Odontologia (CFO). Ofício 447/2020/CFO. Disponível em: http://website.cfo.org.br/wpcontent/uploads/2020/03/oficio-ministro-da-saudecoronavirus-1.pdf. Acessado em: 15/06/2020.

11. Conselho Federal de Medicina. Ofício (CFM) $1456 / 2020$.
Disponível
http://portal.cfm.org.br/images/PDF/2020_oficio_tel emedicina.pdf. Acessado em: 15/06/2020.

12. Conselho Federal de Odontologia (CFO). Resolução CFO-226. Disponível em: http://sistemas. cfo.org.br/visualizar/atos/RESOLUÇÃO/SEC/2020/ 226. Acessado em: 15/06/2020.

13. Conselho Federal de Odontologia (CFO). Código de Ética Odontológica - Resolução CFO-118/2012. Disponível em: http://website.cfo.org.br/wpcontent/uploads/2018/03/codigo_etica.pdf. Acessado em: 15/06/2020.

14. Boringi M, Waghray S, Lavanya R, Babu DBG, Badam RK, Harsha $\mathrm{N}$ et al. Knowledge and Awareness of Teledentistry among Dental Professionals - A Cross Sectional Study. J Clin Diagn Res. 2015;9(8):ZC41-4.

15. Pradhan D, Verma P, Sharma L, Khaitan T. Knowledge, awareness, and attitude regarding teledentistry among postgraduate dental students of Kanpur city, India: A questionnaire study. J Educ Health Promot. 2019;8:104.

16. Cardoso FCC. Utilização de videoconferência nas inspeções anuais de saúde odontológica nas UIS do CPI-4 como forma de priorizar o atendimento dos policiais militares. Dissertação de mestrado. Centro de Altos Estudos de Segurança da Polícia Militar do Estado de São Paulo. 95p. 2019.

17. Paula ADC, Maldonado JMSV, Gadelha CAG. Healthcare telemonitoring and business dynamics: challenges and opportunities for SUS. Rev Saude Publica. 2020;54:65.

18. Fricton J, Chen H. Using teledentistry to improve access to dental care for the underserved. Dent Clin North Am. 2009;53(3):537-48.

19. Cirak B, Kiymaz N, Arslanoglu A. Trigeminal neuralgia caused by intracranial epidermoid tumor: report of a case and review of the different therapeutic modalities. Pain Physician. 2004; $7(1): 129-32$.

20. Marchini L, Ettinger RL. COVID-19 and Geriatric Dentistry: What will be the new-normal? Braz Dent Sci. 2020;23(2):1-7.

\section{CONFLITO DE INTERESSES}

Os autores declaram não haver conflitos de interesse.

\section{AUTOR PARA CORRESPONDÊNCIA}

\section{Thiago Medina Brazoloto}

Rua Dr. Jorge Miranda, 346 - Luz

01106-000 São Paulo - SP, Brasil

E-mail: thibrazoloto@gmail.com 